\title{
Long Non-Coding RNA CBR3-AS1 Promotes Stem- Like Properties and Oxaliplatin Resistance of Colorectal Cancer by Sponging miR-145-5p
}

Liangbao Xie ( $\nabla$ xieliangbaodrmed1@163.com )

The First People's Hospital of Shangqiu https://orcid.org/0000-0003-3087-8458

\section{Guangfei Cui}

The First People's Hospital of Shangqiu

Tao Li

The First People's Hospital of Shangqiu

\section{Primary research}

Keywords: long non-coding RNA CBR3-AS1, colorectal cancer, stem-like properties, oxaliplatin resistance, miR-145-5p

Posted Date: August 17th, 2020

DOI: https://doi.org/10.21203/rs.3.rs-58142/v1

License: (c) (i) This work is licensed under a Creative Commons Attribution 4.0 International License. Read Full License 


\section{Abstract}

Background: Accumulating evidence has shown that long non-coding RNAs (IncRNAs) serve as essential regulators in a plethora of human cancers. In this study, we analyzed the expression profile and functional role of IncRNA CBR3-AS1 in colorectal cancer (CRC).

Methods: CRC tissues and paired adjacent normal tissues were obtained from 133 patients. The expression levels of CBR3-AS1 and miR-145-5p in tissues and cells were detected by RT-qPCR analysis. The proliferation, oxaliplatin resistance, apoptosis, migration, invasion and stem-like properties of CRC cells were detected by MTT assay, flow cytometry analysis, transwell assay and mammosphere formation assay, respectively. Western blot analysis was performed to detect the expression levels of relevant proteins. Dual-luciferase reporter assay and RNA immunoprecipitation assay verified the direct interaction between CBR3-AS1 and miR-145-5p in CRC.

Results: High expression levels of CBR3-AS1 were found in CRC tissues and cell lines. Upregulated CBR3AS1 was closely associated with poor prognosis and adverse clinicopathological features of CRC patients. Artificial knockdown of CBR3-AS1 markedly suppressed the proliferation, migration, invasion and stem-like properties, but promoted the apoptosis of CRC cells. Moreover, we observed that CBR3-AS1 could directly bind to miR-145-5p and negatively regulated its expression in CRC. Further experiments also demonstrated that inhibition of miR-145-5p reverted the effects of CBR3-AS1 knockdown on CRC cells. In addition, compared with the parental cells, CBR3-AS1 expression was strikingly increased in oxaliplatin-resistant CRC cells, and the oxaliplatin resistance was notably diminished by CBR3-AS1 knockdown.

Conclusions: To conclude, our study suggested that CBR3-AS1 serves an oncogenic role in CRC, and may be exploited as a novel therapeutic target for CRC patients.

\section{Background}

Colorectal cancer (CRC) is the third most common cancer and a main cause of cancer-related death worldwide (1). Despite great advancements in diagnostic and therapeutic methods, due to the high frequency of recurrence and metastasis, the long-term prognosis of CRC patients remains largely dismal (2). Therefore, identification of novel therapeutic targets against this fatal malignancy is still urgently needed.

Long non-coding RNAs (IncRNAs) are one group of transcripts with more than 200 nucleotides and deficiency of protein-coding potential. In recent years, IncRNAs have been identified as critical determinants in many physiological and pathological processes, especially in cancer biology $(3,4)$. For example, CBR3-AS1, located on human chromosome 21q22.12, is a recently discovered cancer-related IncRNA. Zhang et al. reported that CBR3-AS1 predicts unfavorable prognosis and promotes tumorigenesis in osteosarcoma (5). Besides, the expression of CBR3-AS1 is also increased in gastric 
cancer tissues (6). In this study, we aimed to detect the expression of CBR3-AS1 in CRC and investigated its functional effects on CRC progression.

\section{Materials And Methods}

\section{Patients and tissue samples}

Primary CRC tissues and paired adjacent non-tumor mucosa tissues were obtained from 133 patients who underwent surgical resection at The First People's Hospital of Shangqiu (Shangqiu, China), and the clinicopathological characteristics of these patients were summarized in Table 1. None of these patients underwent chemotherapy or radiotherapy prior to surgery. After collection, all tissue samples were immediately snap-frozen in liquid nitrogen and stored at $-80^{\circ} \mathrm{C}$.

\section{Cell culture and treatments}

CRC cell lines (HCT116, HT29, SW620 and SW480) and human normal colon epithelial cells (FHC), purchased from American Type Culture Collection (ATCC; Manassas, VA, USA), were cultured in Dulbecco's modified Eagle's medium (DMEM; Thermo Fisher Scientific, Inc., Waltham, MA, USA) containing 10\% fetal bovine serum (FBS; HyClone, Logan, UT, USA) and 1\% penicillin/streptomycin at $37^{\circ} \mathrm{C}$ in a humidified incubator with $5 \% \mathrm{CO}_{2}$.

Oxaliplatin (OXA) was purchased from Meilun Biological Co., Ltd. (Dalian, China). OXA-resistant CRC cell lines (HCT116/OR and SW480/OR cells) were established by exposure to incremental doses of OXA (up to $2 \mu \mathrm{M})$ for 3 months in our laboratory. OXA was removed before the experiments were performed.

The specific small interference RNA (siRNA) targeting CBR3-AS1 (si-CBR3-AS1), siRNA negative control (si-NC), miR-145-5p mimics, miR-145-5p inhibitor, miRNA mimics and inhibitor negative control were synthesized by Guangzhou RiboBio Co., Ltd. (Guangzhou, China). Cells were seeded into 6-well plates one day before transfection and then transfected with the aforementioned molecular products using Lipofectamine 2000 (Invitrogen, Carlsbad, CA, USA). 48 h post-transfection, the transfection efficiency was analyzed by RT-qPCR analysis.

\section{RT-qPCR analysis}

Total RNA was extracted using Trizol reagent (Invitrogen), and quantified using a NanoDrop spectrophotometer (Thermo Fisher Scientific, Inc.). Cytosolic and nuclear fractions of cells were isolated using the NE-PER ${ }^{\text {TM }}$ Nuclear and Cytoplasmic Extraction Reagents Kit (Thermo Fisher Scientific, Inc.). cDNA was synthesized using the PrimeScript RT reagent Kit (TaKaRa, Dalian, China), and PCR amplifications were then carried out using a SYBR Green PCR Kit (TaKaRa) on a 7500HT Real-Time PCR System (Applied Biosystems, Foster City, CA, USA). Data were analyzed using $2^{-\triangle \Delta C t}$ method (7). GAPDH or U6 was used as an internal control.

\section{MTT assay}


Cells were seeded in 96-well plates at a density of 4,000 cells/well. Then, $20 \mu \mathrm{l} \mathrm{MTT}$ solution $(5 \mathrm{mg} / \mathrm{l}$; Sigma-Aldrich, St. Louis, MO, USA) was added to each well. Following incubation for additional $4 \mathrm{~h}, 150$ $\mu$ DMSO (Sigma-Aldrich) was added to dissolve the formazan crystals. Then the absorbance of each well was recorded at $570 \mathrm{~nm}$ and read on a microplate reader (MultiskanEX, Lab systems, Helsinki, Finland).

\section{Cell apoptosis analysis}

Cells were resuspended by $500 \mu \mathrm{l} 1 \times$ binding buffer, and then double-stained with $5 \mu$ l Annexin V-FITC and $5 \mu \mathrm{l} \mathrm{PI} \mathrm{using} \mathrm{an} \mathrm{Annexin-V-PI} \mathrm{Apoptosis} \mathrm{Detection} \mathrm{kit} \mathrm{(BD} \mathrm{Biosciences,} \mathrm{Franklin} \mathrm{Lakes,} \mathrm{NJ,} \mathrm{USA).} \mathrm{The}$ stained cells were then analyzed using a flow cytometry (FACScan; BD Biosciences) equipped with CellQuest software.

\section{Transwell assay}

Cells in serum-free medium were put into the uncoated or matrigel-coated upper chamber of transwell plates ( $8 \mu \mathrm{m}$ pore size; Corning Inc., Corning, NY, USA), while $500 \mu$ l medium containing $10 \%$ FBS was added to the lower chamber. After $24 \mathrm{~h}$, the cells that had not penetrated the membrane were removed using a cotton swab, while the cells on the lower membrane surface were fixed with $75 \%$ methanol and subsequently stained with $0.1 \%$ crystal violet. Then, the stained cells were captured using a microscope (Olympus, Tokyo, Japan).

\section{Mammosphere formation assay}

Single cells prepared by $0.25 \%$ trypsin-EDTA were seeded at a density of 2,000 cells/well in six-well ultralow attachment plates (Corning Inc.), and then cultured with serum-free DMEM/F12 medium (Thermo Fisher Scientific, Inc.) containing $20 \mathrm{ng} / \mathrm{ml}$ human EGF, 1\% B27 and $20 \mathrm{ng} / \mathrm{ml}$ fibroblast growth factor for 7 days. The number of mammospheres $(>20 \mu \mathrm{m})$ was counted under an inverted microscope (Olympus).

\section{Western blot analysis}

Total protein was extracted using RIPA protein extraction reagent (Beyotime, Shanghai, China). Equal amounts of protein samples were separated by SDS-polyacrylamide gel electrophoresis, and transferred to polyvinylidene difluoride membranes (Millipore, Bedford, MA, USA). The membranes were blocked with $5 \%$ skim milk powder for $2 \mathrm{~h}$ at room temperature, followed by incubation with the specific primary antibodies at $4^{\circ} \mathrm{C}$ overnight. The membranes were then incubated with HRP-conjugated secondary antibody at room temperature for $1 \mathrm{~h}$. The blots were visualized using the enhanced chemiluminescence reagents (Bio-Rad Laboratories, Hercules, CA, USA). GAPDH was used as the loading control.

\section{Dual-luciferase reporter assay}

The fragment of CBR3-AS1 containing the predicted miR-145-5p-binding sites was synthesized and inserted into the psiCHECK-2 luciferase reporter vector (Promega, Madison, WI, USA), and mutations were achieved in the binding sites using a Mut Express II Fast Mutagenesis kit (Vazyme, Piscataway, NJ, USA). 
Cells were co-transfected with the luciferase constructs and miR-145-5p mimics or mimics negative control using Lipofectamine 2000. After $48 \mathrm{~h}$ of co-transfection, the luciferase activities were measured using the Dual-Luciferase Reporter Assay System (Promega).

\section{RNA immunoprecipitation (RIP) assay}

RIP assay was performed using the EZ-Magna RIP ${ }^{\mathrm{TM}}$ RNA-Binding Protein Immunoprecipitation Kit (Millipore). The lyase sample of cells transfected with miR-145-5p mimics or mimics negative control was incubated with magnetic beads conjugated with anti-Ago2 or IgG antibody. The immunoprecipitated RNA was purified and subjected to RT-qPCR analysis.

\section{Statistical analysis}

All statistical analyses were performed using GraphPad Prism 6.0 software (GraphPad Software, Inc., La Jolla, CA, USA) and SPSS 18.0 software (SPSS Inc., Chicago, IL, USA). The differences between groups were undertaken using Student's $t$-test or one-way ANOVA followed by Tukey's test. Survival curves were generated by Kaplan-Meier analysis and compared using log-rank test. All $P$ values $<0.05$ were considered to indicate statistical significance.

\section{Results}

\section{CBR3-AS1 is overexpressed in CRC}

First, as indicated by RT-qPCR analysis, CBR3-AS1 was significantly upregulated in CRC tissues, compared with their corresponding normal tissues (Figure 1A). Besides, the expression levels of CBR3AS1 were markedly increased in CRC cell lines (HCT116, HT29, SW620 and SW480), compared to normal FHC cells (Figure 1B). HCT116 and SW480 cells, with the highest expression levels of CBR3-AS1, were selected for further investigation.

We then analyzed the correlation between CBR3-AS1 expression and the clinicopathological parameters of $133 \mathrm{CRC}$ patients. They were allocated into low expression group $(\mathrm{N}=70)$ and high expression group $(\mathrm{N}=63)$, according to the median value of CBR3-AS1 expression. We observed that high CBR3-AS1 expression was closely related to larger tumor size $(P=0.034)$, distant metastasis $(P=0.037)$ and advanced TNM stage ( $P=0.033)$ in CRC patients (Table 1$)$. Moreover, survival analysis showed that CRC patients with high CBR3-AS1 expression had shorter overall survival compared to those with low CBR3AS1 expression ( $P=0.006$; Figure $1 \mathrm{C})$.

\section{CBR3-AS1 knockdown inhibits the malignant behaviors of CRC cells}

To further investigate the biological function of CBR3-AS1 in CRC, HCT116 and SW480 cells were transfected with si-CBR3-AS1. $48 \mathrm{~h}$ after transfection, we found that CBR3-AS1 expression was markedly decreased in both cell lines (Data not shown). As indicated by MTT assay, knockdown of CBR3-AS1 
significantly inhibited the proliferation of HCT116 and SW480 cells (Figure 2A). In addition, as shown in Figure 2B, CBR3-AS1 knockdown triggered the elevation of apoptosis rate in HCT116 and SW480 cells.

Moreover, transwell assay showed that the migration and invasion capacities of HCT116 and SW480 cells were remarkably suppressed when CBR3-AS1 was silenced (Figure 2C). We then cultured HCT116 and SW480 cells in serum-free sphere formation medium, and we found that CBR3-AS1-silenced cells exhibited significantly fewer mammospheres (Figure 2D). The expression levels of CSC markers were detected by western blot analysis. As shown in Figure 2E, CBR3-AS1 knockdown markedly decreased the expression levels of Nanog, Sox2 and Oct4 in HCT116 and SW480 cells.

\section{CBR3-AS1 directly binds to miR-145-5p and inhibits its expression in CRC}

Given that CBR3-AS1 is mainly located in the cytoplasm of HCT116 and SW480 cells (Figure 3A), we therefore hypothesized that it may serve as miRNA sponge in CRC. Through using the Starbase database (http://starbase.sysu.edu.cn/index.php), miR-145-5p was predicted to directly bind to CBR3-AS1, with the binding region shown in Figure 3B. Dual-luciferase reporter assay was further carried out to verify the prediction. As demonstrated in Figure 3C, co-transfection with miR-145-5p mimics markedly decreased the luciferase activity of CBR3-AS1-WT in HCT116 and SW480 cells, rather than CBR3-AS1-MUT. Through RIP assay, we also observed that CBR3-AS1 was strikingly enriched by Ago2 antibody in HCT116 and SW480 cells transfected with miR-145-5p mimics (Figure 3D).

Moreover, Figure 3E indicated that miR-145-5p was significantly downregulated in CRC tissues, and a negative correlation between the expression levels of CBR3-AS1 and miR-145-5p was identified (Figure 3F). In addition, the expression of miR-145-5p was significantly increased by si-CBR3-AS1 in HCT116 and SW480 cells (Figure 3G).

\section{miR-145-5p inhibition blocks the effects of CBR3-AS1 knockdown in CRC cells}

MTT assay further showed that co-transfection with miR-145-5p inhibitor significantly rescued the impaired proliferation of HCT116 and SW480 cells after CBR3-AS1 knockdown (Figure 4A), and the increased apoptosis rates of these cells were also diminished by miR-145-5p inhibition (Figure 4B). Moreover, the inhibitory effects of si-CBR3-AS1 on the migration and invasion of HCT116 and SW480 cells were markedly blocked by miR-145-5p inhibition (Figure 4C). We further noticed that miR-145-5p inhibition increased the number of cell mammospheres (Figure 4D), accompanied by the increased expression levels of Nanog, Sox2 and Oct4 (Figure 4E).

\section{CBR3-AS1 knockdown blocks OXA resistance in CRC cells}

We then established OXA-resistant CRC cell lines, and MTT assay confirmed that, compared with the parental cells, HCT116/OR and SW480/OR cells showed more resistance to OXS (Figure 5A). We also observed that the expression levels of CBR3-AS1 were strikingly increased, while miR-145-5p was downregulated in HCT116/OR and SW480/OR cells (Figure 5B-C). Furthermore, as shown in Figure 5D-E, 
CBR3-AS1 knockdown significantly enhanced the chemosensitivity of HCT116/OR and SW480/OR cells to OXA, but this effect was notably diminished by miR-145-5p inhibition.

\section{Discussion}

$\mathrm{CRC}$ is a heterogeneous multifactorial disease. Overwhelming evidence has demonstrated that numerous IncRNAs are closely associated with CRC progression and development (8). They have various expression patterns in CRC samples, and can serve as oncogenes or tumor suppressors. Targeting IncRNAs and elucidating the underlying mechanisms may improve the efficacies of diagnostic and therapeutic methods for CRC.

In this study, CBR3-AS1 was found to be upregulated in CRC tissues and cell lines, and it was closely correlated with poor prognosis and adverse clinicopathological features of CRC patients. By loss-offunction assays, we further observed that CBR3-AS1 knockdown yielded significant inhibitory effects on the malignant properties of CRC cells. OXA is a first-line chemotherapeutic drug for CRC patients, but chemoresistance remains a major challenge (9). Cancer stem cells (CSCs) play a critical role in the recurrence and chemoresistance of CRC, and targeting self-renewal of CSCs may represent a new paradigm in CRC therapy $(10,11)$. This study also indicated that CBR3-AS1 knockdown suppressed stemlike properties and oxaliplatin resistance of CRC cells.

As a class of short ( $22 \mathrm{nt})$ non-coding RNAs, microRNAs (miRNAs) also significantly contribute to CRC progression and development (12). Up to now, accumulating articles indicated that IncRNAs located in the cytoplasm always function as molecular sponges to compete miRNAs in a sequence-specific manner (13). miR-145-5p was previously reported to be a tumor suppressor in many cancers, including CRC (14, 15), and in our study, we confirmed that CBR3-AS1 could directly bind to miR-145-5p and suppressing its expression in CRC. Compared with the parental cells, miR-145-5p expression was also decreased in OXAresistant CRC cells. Furthermore, rescue experiments showed that miR-145-5p inhibition blocked the effects of CBR3-AS1 knockdown in CRC cells.

\section{Conclusions}

In conclusion, the present study provided convincing evidence that CBR3-AS1 promotes stem-like properties and OXA resistance of CRC cells partly by sponging miR-145-5p. CBR3-AS1 could be considered as a potential therapeutic target for CRC patients in the future.

\section{Declarations}

\section{Declarations}

Ethics approval and consent to participate 
This study was approved by the Ethics Committee of The First People's Hospital of Shangqiu. Written informed consent was obtained from all subjects in accordance with the Declaration of Helsinki.

Consent for publication

Not applicable.

Availability of data and material

All data generated or analyzed during this study are included in this article.

Competing interests

The authors declare that they have no competing interests.

\section{Funding}

Not applicable.

\section{Authors' contributions}

Liangbao Xie conceived the study and designed the experiments. Liangbao Xie, Guangfei Cui and Tao Li performed the experiments and analyzed the data. Liangbao Xie supervised the study and wrote the manuscript. Guangfei Cui and Tao Li reviewed the manuscript. All authors read and approved the final manuscript.

\section{Acknowledgements}

Not applicable.

\section{References}

1. Anvarnia A, Mohaddes-Gharamaleki F, Asadi M, Akbari M, Yousefi B, Shanehbandi D. Dysregulated microRNAs in colorectal carcinogenesis: New insight to cell survival and apoptosis regulation. Journal of cellular physiology. 2019;234(12):21683-93.

2. Brouwer NPM, Bos A, Lemmens V, Tanis PJ, Hugen N, Nagtegaal ID, et al. An overview of 25 years of incidence, treatment and outcome of colorectal cancer patients. International journal of cancer. 2018;143(11):2758-66.

3. Spizzo R, Almeida MI, Colombatti A, Calin GA. Long non-coding RNAs and cancer: a new frontier of translational research? Oncogene. 2012;31(43):4577-87.

4. Yang G, Lu X, Yuan L. LncRNA: a link between RNA and cancer. Biochim Biophys Acta. 2014;1839(11):1097-109. 
5. Zhang Y, Meng W, Cui H. LncRNA CBR3-AS1 predicts unfavorable prognosis and promotes tumorigenesis in osteosarcoma. Biomedicine pharmacotherapy = Biomedecine pharmacotherapie. 2018;102:169-74.

6. Gao J, Cao R, Mu H. Long non-coding RNA UCA1 may be a novel diagnostic and predictive biomarker in plasma for early gastric cancer. Int J Clin Exp Pathol. 2015;8(10):12936-42.

7. Livak KJ, Schmittgen TD. Analysis of relative gene expression data using real-time quantitative PCR and the 2(-Delta Delta C(T)) Method. Methods. 2001;25(4):402-8.

8. Kim T, Croce CM. Long noncoding RNAs: Undeciphered cellular codes encrypting keys of colorectal cancer pathogenesis. Cancer letters. 2018;417:89-95.

9. Martinez-Balibrea E, Martinez-Cardus A, Gines A, Ruiz de Porras V, Moutinho C, Layos L, et al. TumorRelated Molecular Mechanisms of Oxaliplatin Resistance. Mol Cancer Ther. 2015;14(8):1767-76.

10. Zeuner A, Todaro M, Stassi G, De Maria R. Colorectal cancer stem cells: from the crypt to the clinic. Cell stem cell. 2014;15(6):692-705.

11. Kreso A, van Galen P, Pedley NM, Lima-Fernandes E, Frelin C, Davis T, et al. Self-renewal as a therapeutic target in human colorectal cancer. Nature medicine. 2014;20(1):29-36.

12. Ding $L$, Lan Z, Xiong $X$, Ao H, Feng $Y, G u H$, et al. The Dual Role of MicroRNAs in Colorectal Cancer Progression. International journal of molecular sciences. 2018;19(9).

13. Ebert MS, Sharp PA. Emerging roles for natural microRNA sponges. Current biology: CB. 2010;20(19):R858-61.

14. Niu Y, Zhang J, Tong Y, Li J, Liu B. miR-145-5p restrained cell growth, invasion, migration and tumorigenesis via modulating RHBDD1 in colorectal cancer via the EGFR-associated signaling pathway. Int J Biochem Cell Biol. 2019;117:105641.

15. Chen Q, Zhou L, Ye X, Tao M, Wu J. miR-145-5p suppresses proliferation, metastasis and EMT of colorectal cancer by targeting CDCA3. Pathol Res Pract. 2020;216(4):152872.

\section{Tables}

Table 1 The relationship between clinicopathological characteristics and CBR3-AS1 expression in 133 CRC patients. 


\begin{tabular}{|c|c|c|c|c|}
\hline \multirow[t]{2}{*}{ Characteristics } & \multirow{2}{*}{$\begin{array}{l}\text { Total number } \\
(\mathrm{N}=133)\end{array}$} & \multicolumn{2}{|c|}{ CBR3-AS1 expression } & \multirow[t]{2}{*}{$P$ value } \\
\hline & & Low $(\mathrm{N}=70)$ & High $(\mathrm{N}=63)$ & \\
\hline Age (years) & & & & 0.236 \\
\hline$<60$ & 47 & 28 & 19 & \\
\hline$\geq 60$ & 86 & 42 & 44 & \\
\hline Gender & & & & 0.455 \\
\hline Male & 80 & 40 & 40 & \\
\hline Female & 53 & 30 & 23 & \\
\hline Tumor size $(\mathrm{cm})$ & & & & 0.034 \\
\hline$<5$ & 74 & 45 & 29 & \\
\hline$\geq 5$ & 59 & 25 & 34 & \\
\hline Tumor differentiation & & & & 0.176 \\
\hline Well+Moderate & 100 & 56 & 44 & \\
\hline Poor & 33 & 14 & 19 & \\
\hline Lymph node metastasis & & & & 0.215 \\
\hline No & 56 & 33 & 23 & \\
\hline Yes & 77 & 37 & 40 & \\
\hline Distant metastasis & & & & 0.037 \\
\hline No & 84 & 50 & 34 & \\
\hline Yes & 49 & 20 & 29 & \\
\hline TNM stage & & & & 0.033 \\
\hline I-II & 72 & 44 & 28 & \\
\hline III-IV & 61 & 26 & 35 & \\
\hline
\end{tabular}

Figures 
A

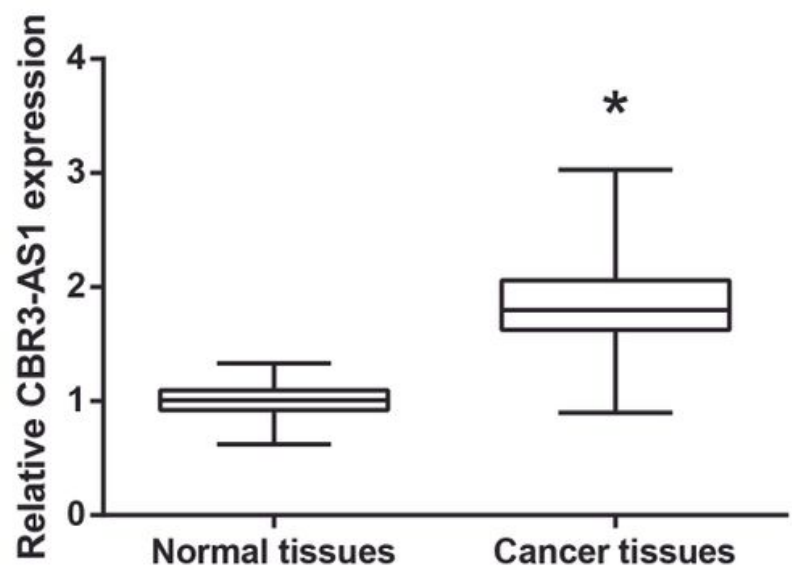

B

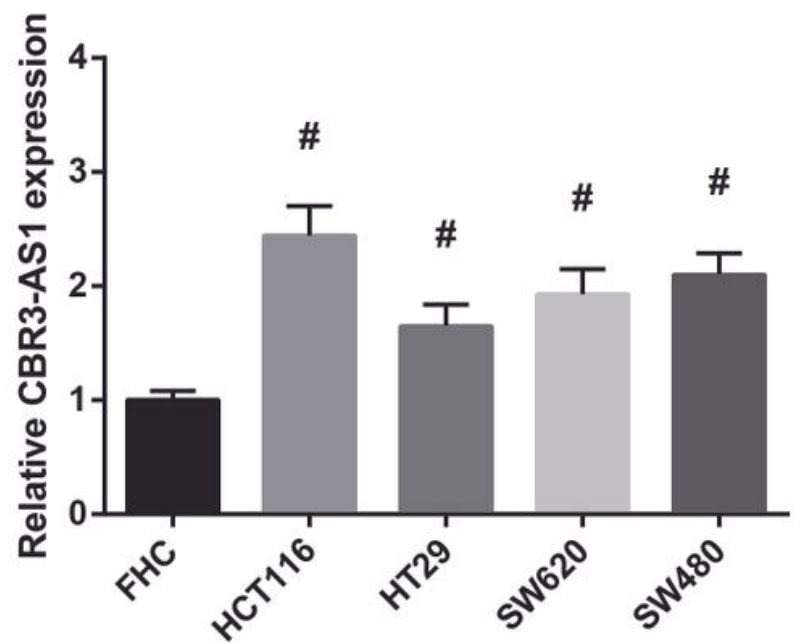

C

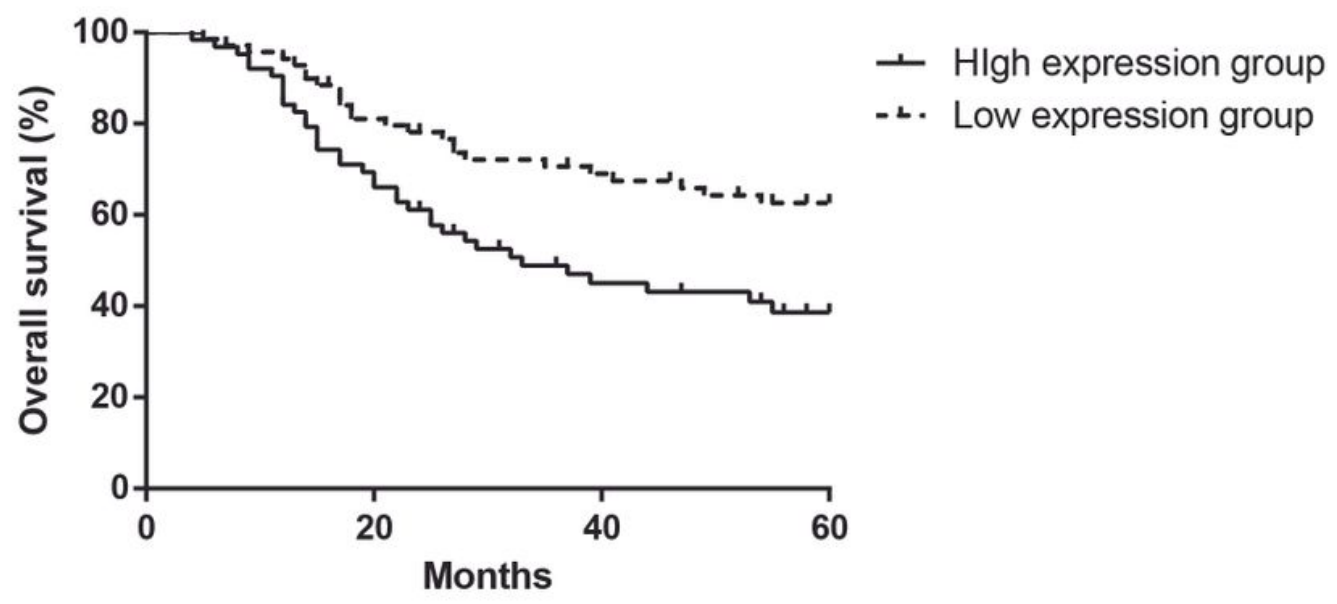

Figure 1

CBR3-AS1 is overexpressed in CRC. (A) RT-qPCR analysis of CBR3-AS1 expression levels in CRC tissues and adjacent normal tissues. (B) RT-qPCR analysis of CBR3-AS1 expression levels in CRC cell lines and FHC cells. (C) Kaplan-Meier analysis of correlation between CBR3-AS1 expression and overall survival of CRC patients. ${ }^{*}<<0.05$ vs. normal tissues; $\# P<0.05$ vs. $F H C$ cells. 
A
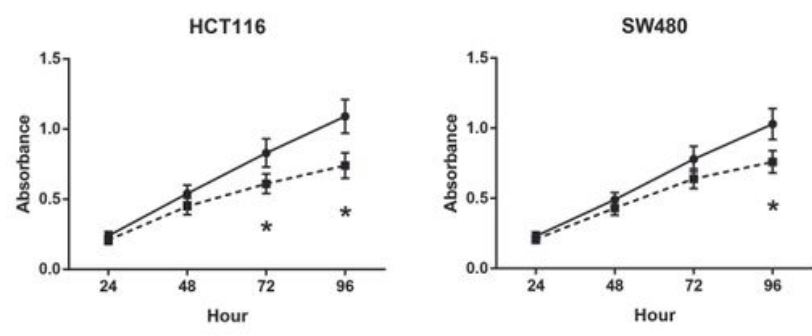

-. si-CBR3-AS1

$\rightarrow$ si-NC
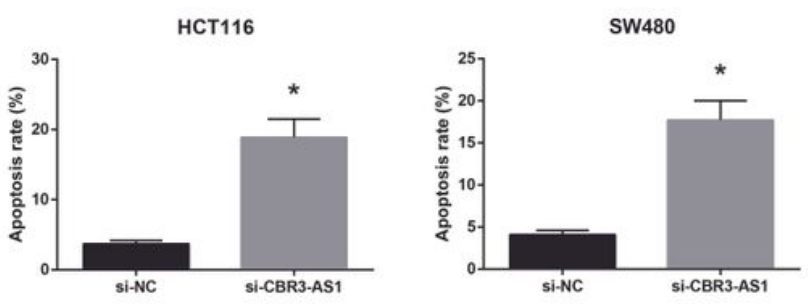

C
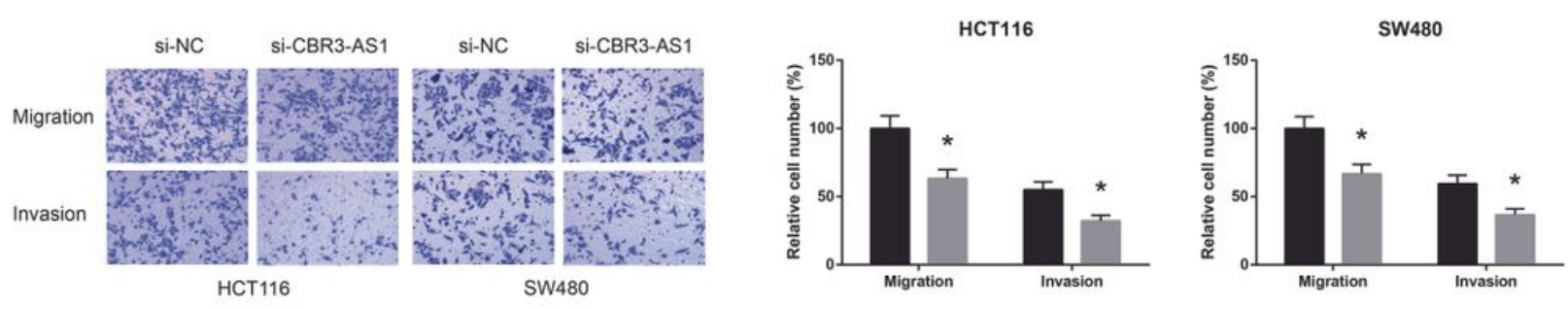

- si-NC si-CBR3-AS

D
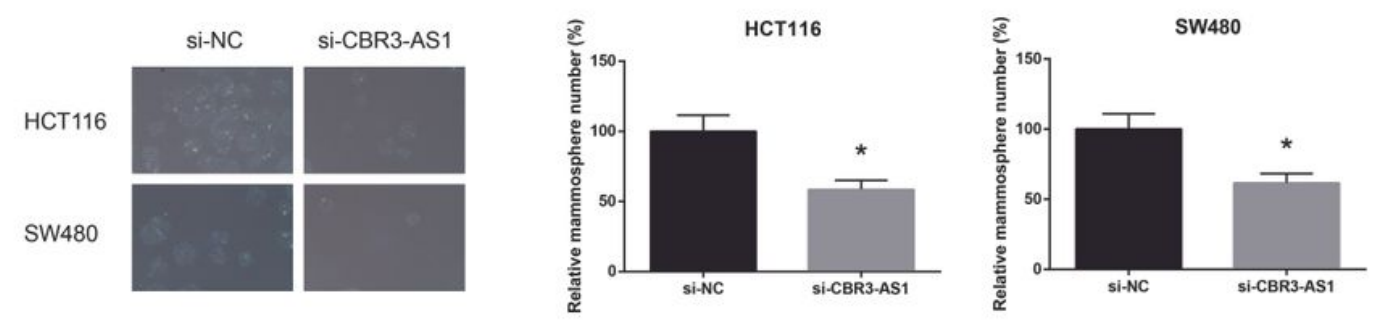

$\mathrm{E}$
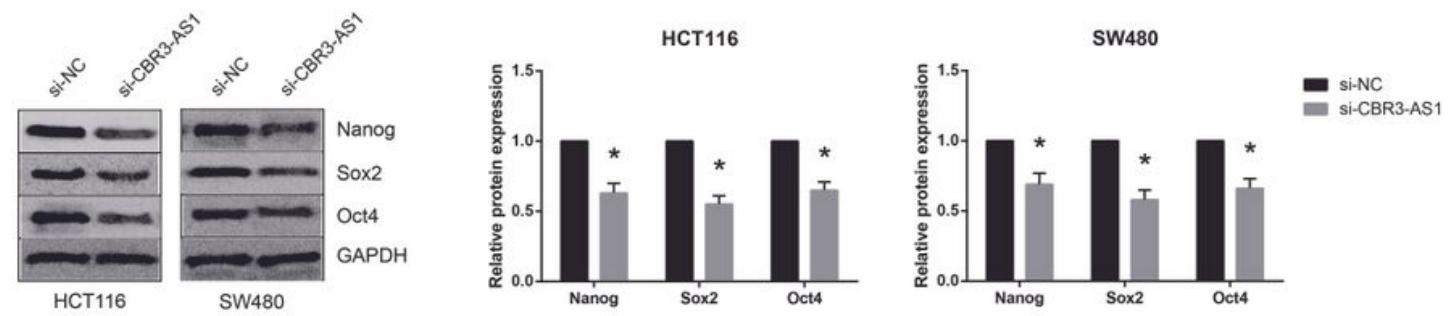

Figure 2

CBR3-AS1 knockdown inhibits the malignant behaviors of CRC cells. (A) MTT assay showed the proliferation of CRC cells after transfection. (B) Flow cytometry analysis showed the apoptosis of CRC cells after transfection. (C) Transwell assay showed the migration and invasion of CRC cells after transfection. (D) Mammosphere formation assay showed the number of CRC cell mammospheres after transfection. (E) Western blot analysis showed the expression levels of CSC markers in CRC cells after transfection. ${ }^{*} \mathrm{P}<0.05$ vs. si-NC-transfected cells. 
A

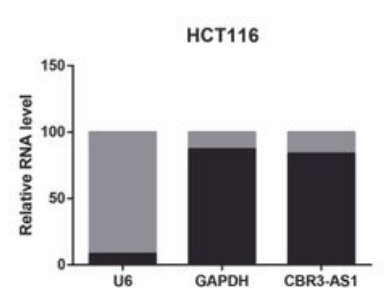

C

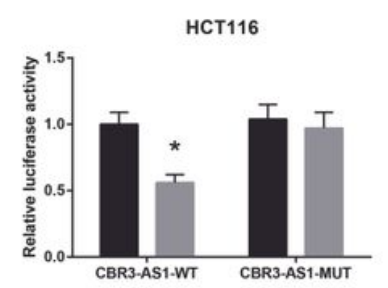

E

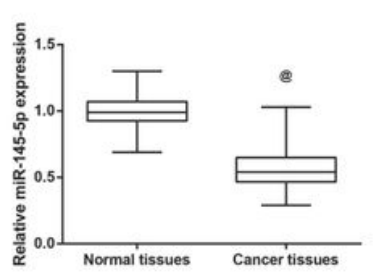

sW480

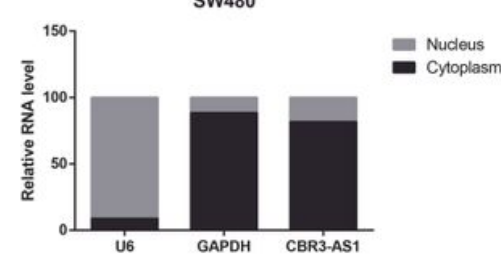

SW480

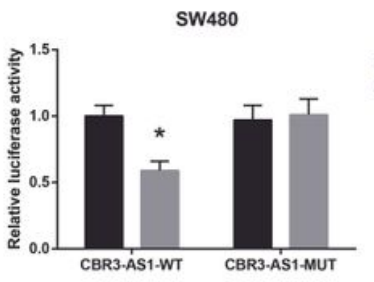

$\mathrm{F}$

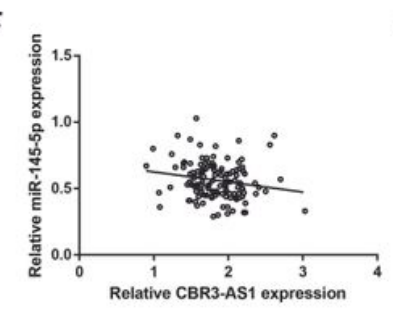

G
B

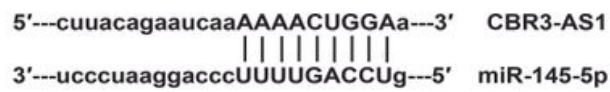

D
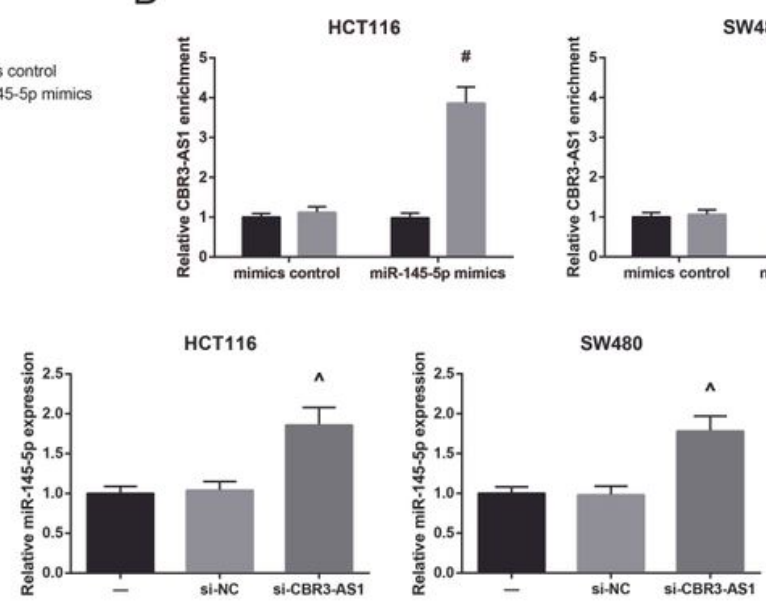

Figure 3

CBR3-AS1 directly binds to miR-145-5p and inhibits its expression in CRC. (A) Cell fractionation analysis showed the subcellular distribution of CBR3-AS1 in CRC cells. (B) The predicted binding site of miR-145$5 p$ within CBR3-AS1 fragment. (C) Dual-luciferase reporter assay validated the binding relation between CBR3-AS1 and miR-145-5p in CRC cells. (D) RIP assay showed the enrichment of CBR3-AS1 in CRC cells after transfection. (E) RT-qPCR analysis of miR-145-5p expression levels in CRC tissues and adjacent normal tissues. (F) An inverse expression correlation between CBR3-AS1 and miR-195-5p in CRC tissues. (G) RT-qPCR analysis of miR-145-5p expression levels in CRC cells after transfection. ${ }^{*} P<0.05$ vs. mimics control-transfected cells; \#P<0.05 vs. IgG antibody; @P $<0.05$ vs. normal tissues; ${ }^{\wedge} \mathrm{P}<0.05$ vs. si-NCtransfected cells. 
A

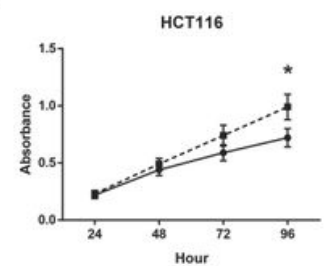

C

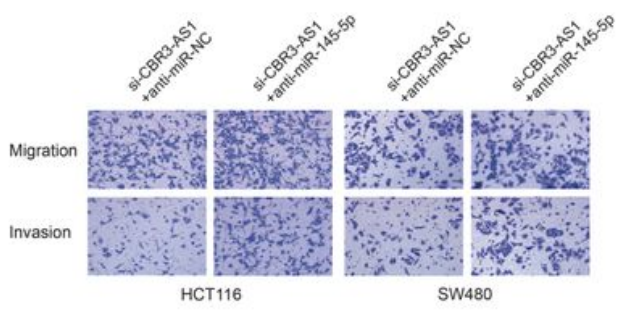

SW480

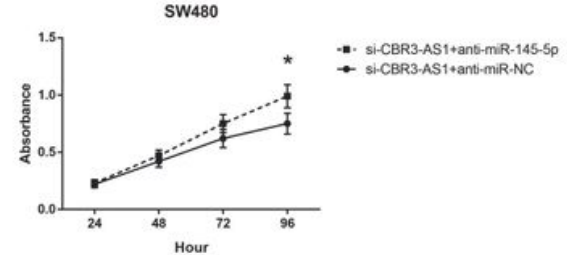

B
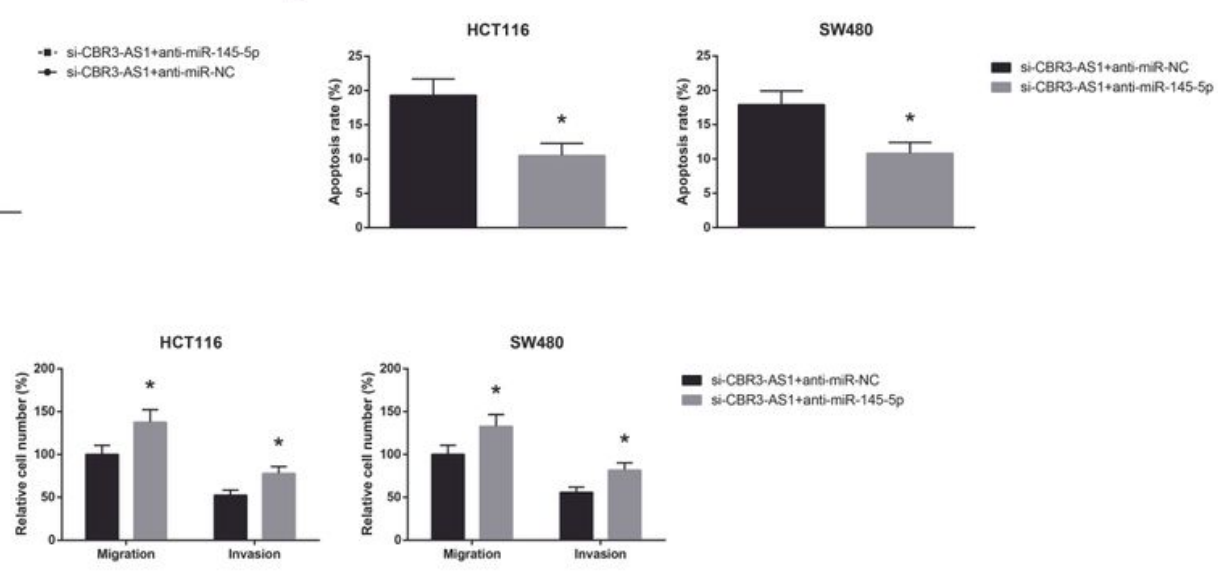

D
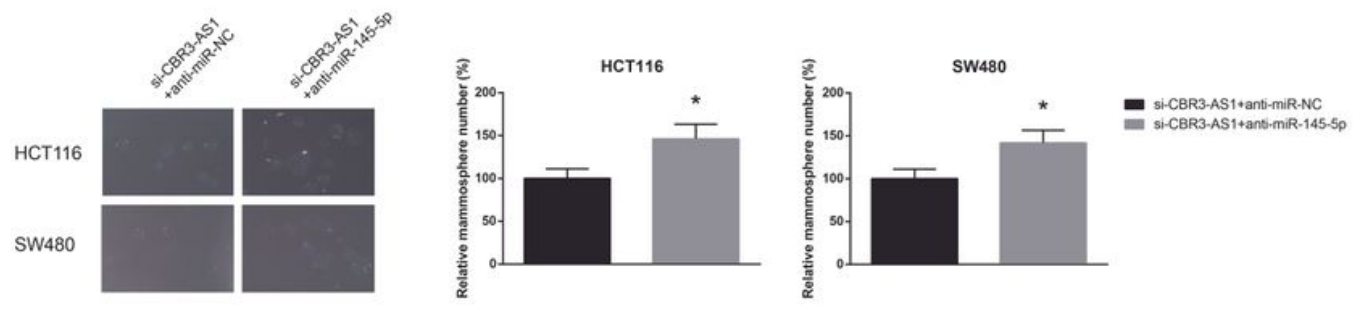

E
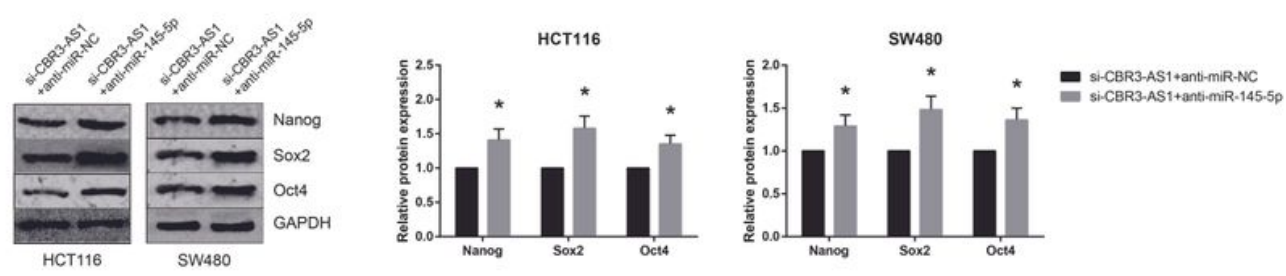

\section{Figure 4}

miR-145-5p inhibition blocks the effects of CBR3-AS1 knockdown in CRC cells. (A) MTT assay showed the proliferation of CRC cells after transfection. (B) Flow cytometry analysis showed the apoptosis of CRC cells after transfection. (C) Transwell assay showed the migration and invasion of CRC cells after transfection. (D) Mammosphere formation assay showed the number of CRC cell mammospheres after transfection. (E) Western blot analysis showed the expression levels of CSC markers in CRC cells after transfection. ${ }^{*} \mathrm{P}<0.05$ vs. NC inhibitor-transfected cells. 
A
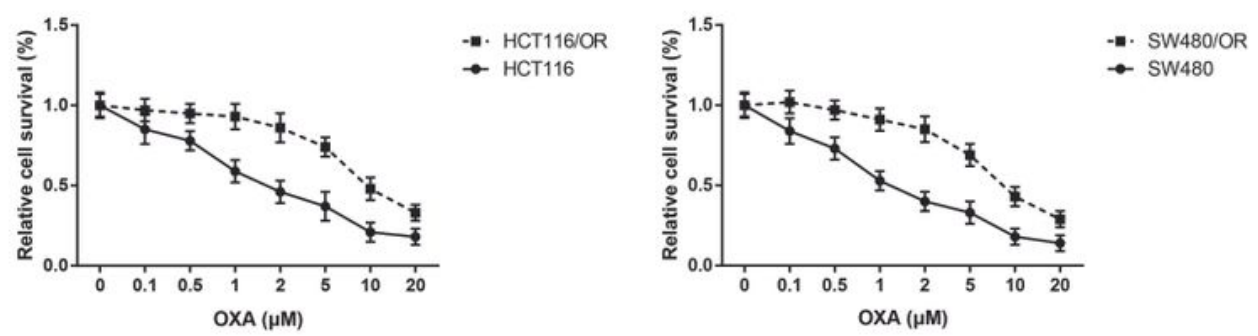

B
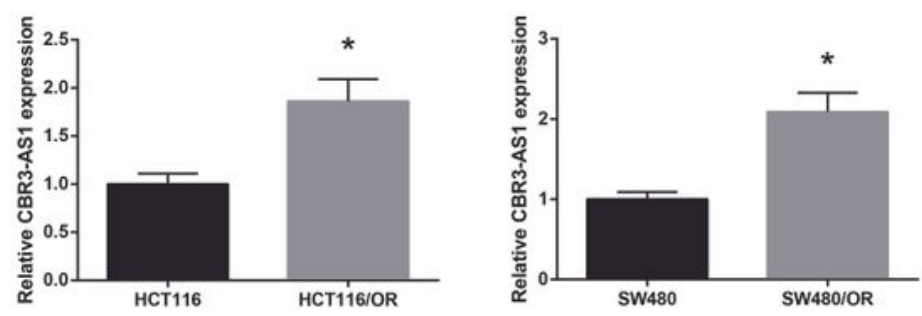

C
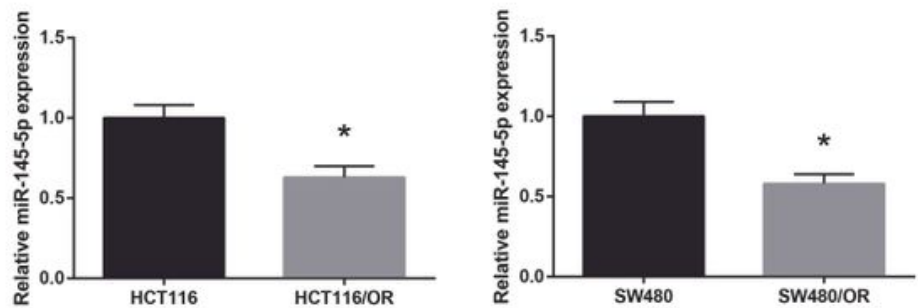

D
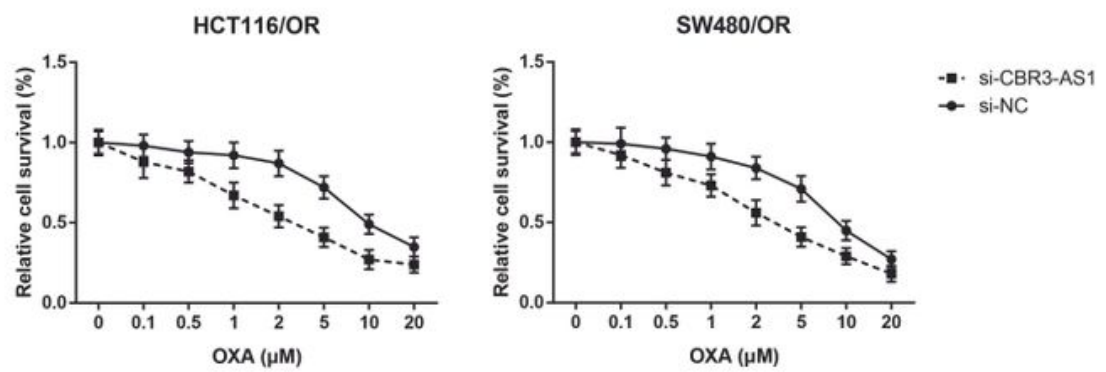

$E$
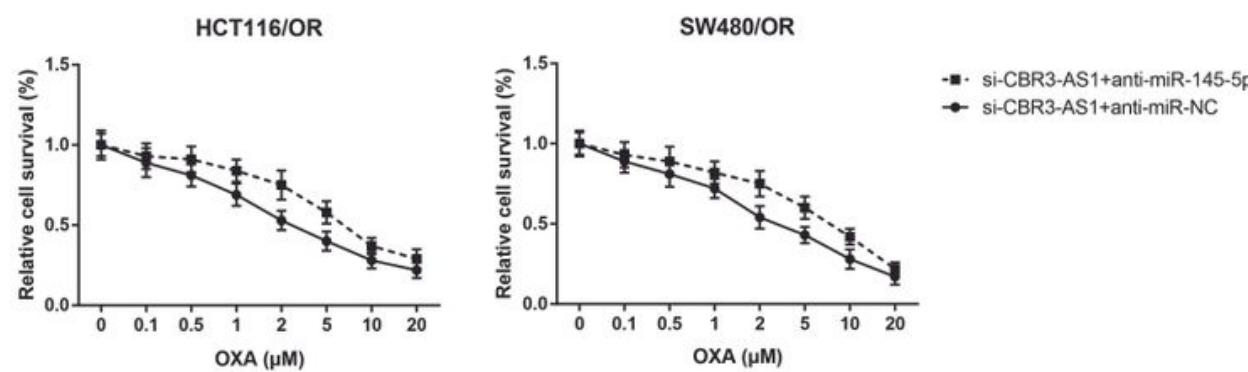

\section{Figure 5}

CBR3-AS1 knockdown blocks OXA resistance in CRC cells. (A) MTT assay showed the chemosensitivity of OXA-resistant CRC cells and parental cells to OXA. (B) RT-qPCR analysis of CBR3-AS1 expression levels in OXA-resistant CRC cells and parental cells. (C) RT-qPCR analysis of miR-145-5p expression levels in OXA-resistant CRC cells and parental cells. (D-E) MTT assay showed the chemosensitivity of OXAresistant CRC cells to OXA after transfection. ${ }^{\star} \mathrm{P}<0.05$ vs. parental cells. 